\title{
A Multi-institutional Analysis of Perioperative Outcomes in 106 Men Who Underwent Radical Prostatectomy for Distant Metastatic Prostate Cancer at Presentation
}

\author{
Prasanna Sooriakumaran $^{a, b}$, Jeffrey Karnes $^{c}$, Christian Stief $^{d}$, Bethan Copsey ${ }^{e}$, \\ Francesco Montorsi $^{f}$, Peter Hammerer ${ }^{g}$, Burkhard Beyer $^{h}$, Marco Moschini $^{c}$, \\ Christian Gratzke ${ }^{d}$, Thomas Steuber ${ }^{h}$, Nazareno Suardi $^{f}$, Alberto Briganti $^{f}$, Lukas Manka $^{g}$, \\ Tommy Nyberg $^{b}$, Susan J. Dutton ${ }^{e}$, Peter Wiklund ${ }^{b, i}$, Markus Graefen ${ }^{h, *}$ \\ ${ }^{a}$ Nuffield Department of Surgical Sciences, University of Oxford, Oxford, UK; ${ }^{\mathrm{b}}$ Department of Molecular Medicine and Surgery, Karolinska Institutet, \\ Stockholm, Sweden; ${ }^{\mathrm{c}}$ Mayo Clinic Department of Urology, Rochester, USA; ${ }^{\mathrm{d}}$ Department of Urology, Ludwig Maximilian University of Munich, Munich, \\ Germany; ${ }^{\mathrm{e}}$ Centre for Statistics in Medicine, University of Oxford, Oxford, UK; ${ }^{\mathrm{f}}$ Department of Urology, San Raffaele Hospital, Milan, Italy; ${ }^{\mathrm{g}}$ Department of \\ Urology/Uro-oncology, Academic Hospital Braunschweig, Brunswick, Germany; ${ }^{\mathrm{h}}$ Martini Clinic, Prostate Cancer Center, University Hospital Hamburg- \\ Eppendorf, Hamburg, Germany; ${ }^{\mathrm{i}}$ Uro-clinic, St. Goran Hospital, Stockholm, Sweden
}

\section{Article info}

Article history:

Accepted May 14, 2015

Associate Editor:

James Catto

\section{Keywords:}

Metastatic prostate cancer

Radical prostatectomy

Surgery

Safety

Perioperative outcomes

\begin{abstract}
Background: Current trials are investigating radical intervention in men with metastatic prostate cancer. However, there is a lack of safety data for radical prostatectomy as therapy in this setting.

Objective: To examine perioperative outcomes and short-term complications after radical prostatectomy for locally resectable, distant metastatic prostate cancer.

Design, setting, and participants: A retrospective case series from 2007 to 2014 comprising 106 patients with newly diagnosed metastatic (M1) prostate cancer from the USA, Germany, Italy, and Sweden.

Intervention: Radical prostatectomy and extended pelvic lymphadenectomy.

Outcome measurements and statistical analysis: Descriptive statistics were used to present margin status, continence, and readmission, reoperation, and overall complication rates at $90 \mathrm{~d}$, as well as for 21 specific complications. Kaplan-Meier plots were used to estimate survival function. Intercenter variability and M1a/ M1b subgroups were examined.

Results and limitations: Some $79.2 \%$ of patients did not suffer any complications; positive-margin (53.8\%), lymphocele ( $8.5 \%$ ), and wound infection $(4.7 \%)$ rates were higher in our cohort than in a meta-analysis of open radical prostatectomy performed for standard indications. At a median follow-up of $22.8 \mathrm{mo}, 94 / 106(88.7 \%)$ men were still alive. The study is limited by its retrospective design, differing selection criteria, and short follow-up.

Conclusions: Radical prostatectomy for men with locally resectable, distant metastatic prostate cancer appears safe in expert hands for meticulously selected patients. Overall and specific complication rates related to the surgical extirpation are not more frequent
\end{abstract}

\footnotetext{
* Corresponding author. Martini Clinic, Prostate Cancer Center, University Hospital HamburgEppendorf, Martinistrasse 52, 20246 Hamburg. Germany. Tel. +40 7410-51314; Fax: +040-7410-51323.

E-mail address: graefen@uke.uni-hamburg.de (M. Graefen).
}

http://dx.doi.org/10.1016/j.eururo.2015.05.023

0302-2838/C 2015 Published by Elsevier B.V. on behalf of European Association of Urology. 
than when radical prostatectomy is performed for standard indications, and the use of extended pelvic lymphadenectomy in all of this cohort compared to its selective use in localized/locally advanced prostate cancer accounts for any extra morbidity.

Patient summary: Men presenting with advanced prostate cancer that has spread beyond the prostate are increasingly being considered for treatments directed at the prostate itself. On the basis of results for our international series of 106 men, surgery appears reasonably safe in this setting for certain patients.

(C) 2015 Published by Elsevier B.V. on behalf of European Association of Urology.

\section{Introduction}

Metastatic prostate cancer is a major source of morbidity and mortality, and survival rates remain poor [1,2]. In fact, a recent UK-wide trial has demonstrated median overall survival of 42 mo in men with newly diagnosed metastatic prostate cancer treated with initial systemic therapy alone [3]. Recent interest in considering radical intervention in the management of metastatic disease has surfaced [4], and there is a precedent for such an approach for other tumor types. European Organisation for Research and Treatment of Cancer and Southwest Oncology Group studies have transformed the management of metastatic renal cell cancer by demonstrating an improvement in survival of $13-26 \%$ after nephrectomy plus systemic therapy compared to systemic therapy alone [5,6]. A meta-analysis of 6885 women with advanced ovarian carcinoma found a median survival of 33.9 mo in those treated with $>75 \%$ maximal cytoreductive surgery versus 22.7 mo for those with $\leq 25 \%$ cytoreduction [7]. A recent Surveillance, Epidemiology, and End Results study of 8185 men with metastatic prostate cancer revealed higher 5-yr overall and cancer-specific survival rates for the surgical and brachytherapy cohorts than for patients who received initial androgen deprivation therapy (ADT) alone [8]. These data were backed up results from the Munich Cancer Registry for 74 men with metastatic cancer treated with radical prostatectomy and 1464 treated with initial ADT alone; the 5-yr overall survival was 55\% in the surgical group versus $21 \%$ in the ADT group [9]. This series also demonstrates that radical intervention therapies have been used in some men with metastatic prostate cancer at presentation in the absence of a clear rationale. In fact, a recent, large, observational, population-based study in Sweden suggested that rather large numbers of such cases exist [10].

As well as the epidemiologic data outlined above, there is a biologic basis for considering radical intervention in men with metastatic prostate cancer. Paget's "seed and soil" hypothesis states that a receptive microenvironment (the "soil") is required, into which disseminating cancer cells (the "seeds") can engraft and form metastases; it may be that "soil" development is driven by hematopoietic and other factors secreted by the primary tumor [11]. Therefore, development of individual metastases might conceivably be dependent on an intact primary focus, giving credence to the hypothesis that intervention targeting the primary lesion can impact metastatic progression and delay death. The above data are certainly not conclusive, and there is evidence of the converse as well, that surgery can actually promote tumor growth, invasion, or angiogenesis via a variety of wound healing-induced growth factors, chemokines, and cytokines [12]. Investigators have recently demonstrated that primary tumor clones from prostate cancer patients are still present several years after radical prostatectomy [13], and that metastases themselves can drive their own progression, possibly without a primary tumor acting as the stimulus [14]. Hence, the situation is complex and the hypothesis that radical therapy can positively impact survival in metastatic prostate cancer needs better evaluation. As a result, investigators started interrogation with randomized controlled trials; the UK STAMPEDE (www. clinicaltrials.gov NCT00268476) and Dutch HORRAD (www. trialregister.nl NTR271) trials are examining the role of radical intervention in metastatic prostate cancer using radiation as the modality of choice. The data outlined above, however, suggest that surgery is also worthy of investigation as a radical intervention in patients with metastatic disease, but only one trial is currently doing this. An MD Anderson Cancer Center study is giving patients with metastatic disease who respond to systemic therapy the choice of surgery versus radiation as local therapy (www.clinicaltrials. gov NCT01751438) [15]. Although it has been shown that surgery is safe in men with localized disease [16], the concern is that it might not be feasible or safe in patients with metastatic disease, which is why study investigators have focused on radiation therapy as the radical modality of choice. Here we address the safety of radical prostatectomy in men with distant metastases to inform future trials considering primary-directed therapy using surgery in advanced prostate cancer.

\section{Patients and methods}

Patients included in the cohort study were men who underwent radical prostatectomy for known distant metastatic prostate cancer at presentation between 2007 and 2014. Data were collected in a nontrial setting from six centers in the USA, Germany, Italy, and Sweden. No welldefined selection criteria were applied and patients were selected according to the clinician's subjective judgment of whether they were predicted to benefit from the procedure and whether the cancer seemed locally resectable. For some cases, the clinician judgment favored surgery because of local symptoms such as pain or obstruction, whereas for others surgery was performed on the basis of the experimental oncologic rationale discussed above [8-11]. All surgeons performed what they self-termed an extended pelvic lymphadenectomy at the time of the surgery to include the obturator, external iliac, and common iliac nodes up to the ureteral crossing. However, the template was not standardized among centers, and in some cases the internal iliac, fossa of Marcille, and/or presacral nodes were also included. The choice of surgical modality between open or robotic was also left to individual surgeon discretion. Outcome data provided also varied among centers because of differing practices for recording, but generally covered patient characteristics, clinically relevant measures, features of the 
surgical procedure, complications, and adjuvant/salvage treatments. Specific variables recorded included age; body mass index; Charlson comorbidity index (CCI); prostate volume; Gleason score; tumor stage according to the TNM system (although staging modalities were not standardized among centers); prostate-specific antigen (PSA); continence (number of pads per $24 \mathrm{~h}$ ); operative modality; operative time; number of lymph nodes removed; margin status; length of hospital stay; catheterization duration; types of neoadjuvant, adjuvant and salvage therapies; and complications according to the meta-analysis of Tewari et al [16]. The complications assessed included reoperation, readmission, transfusion, intraoperative injury (vessel, obturator nerve, ureteral, bladder, rectal, other bowel, need for ileostomy), ileus, deep venous thrombosis, pulmonary embolism, pneumonia, myocardial infarction, hematoma, lymphocele, anastomotic leak, anastomotic stricture, sepsis, wound infection, and wound dehiscence. As only 6/106 (5.7\%) of men underwent nerve-sparing surgery on the basis of intraoperative results for frozen sections in one center, pre- and postoperative potency data were not captured. All centers recorded deaths and cause of death, as well as time from surgery until last follow-up. For data not recorded prospectively, case notes were abstracted by a member of the center's surgical team and records were updated accordingly.

\subsection{Statistical methods}

Data were analyzed using STATA IC 13 (StataCorp, College Station, TX, USA) and exploratory analysis consisted primarily of descriptive statistics. The median and interquartile range (IQR) are reported for continuous variable, and the frequency and percentage for dichotomous and categorical variables. When considered appropriate, results are presented by center to account for differences in surgical procedure and postoperative patient care. Reverse Kaplan-Meier analysis techniques were used to assess follow-up times, with censoring for deaths, and Kaplan-Meier plots demonstrated survival functions. Subgroup analyses were performed to compare M1a (extrapelvic nodal disease) and M1b (skeletal metastases) cases in terms of differences in patient characteristics and radical prostatectomy results.

\section{Results}

For the 106 men in the study, Table 1 shows important patient and tumor characteristics. The median PSA was $23.5 \mathrm{ng} / \mathrm{ml}$ (IQR 8.1-55.1). CCI was recorded for 75/106 (70.8\%) cases: 58/75 (77.3\%) were CCI 0, and 17/75 (22.7\%) were CCI 1 (Table 2). Some 39/106 (36.8\%) men had T3/4 disease and 45/106 (42.5\%) had positive nodes before surgery. Among the patients, 70/106 (66.0\%) had skeletal metastases: the number of skeletal lesions was not recorded in $34 / 70$ (48.6\%), one lesion in 20/70 (28.6\%), two lesions in $7 / 70$ (10.0\%), and $\geq 3$ lesions in $9 / 70$ (12.9\%) cases. Four
Table 1 - Baseline characteristics for the whole cohort $(n=106)$

\begin{tabular}{lc}
\hline Parameter & Value \\
\hline Age $(\mathrm{yr})$ & $64.5(58.0-70.0)$ \\
Body mass index $\left(\mathrm{kg} / \mathrm{m}^{2}\right)^{\mathrm{a}}$ & $26.9(24.7-28.7)$ \\
Prostate volume $\left(\mathrm{cm}^{3}\right)^{\mathrm{b}}$ & $40(29.6-58.2)$ \\
PSA $(\mathrm{ng} / \mathrm{ml})$ & $23.5(8.1-55.1)$ \\
Gleason score & $8.5(8.0-9.0)$ \\
\hline Data are presented as median (interquartile range). & \\
a $n=101$. & \\
$\mathrm{b}$ & $n=100$. \\
\hline
\end{tabular}

Table 2 - Preoperative comorbidity scores and staging for the whole cohort

\begin{tabular}{|cc}
\hline & $n(\%)$ \\
\hline Charlson comorbidity index & \\
0 & $58(77.3)$ \\
\hline 1 & $17(22.7)$ \\
\hline Preoperative T stage & \\
\hline T0/Tx & $42(39.6)$ \\
T2 & $25(23.6)$ \\
\hline T3a & $17(16.0)$ \\
T3b & $13(12.3)$ \\
\hline T4 & $9(8.5)$ \\
\hline Preoperative N stage & \\
\hline N0/Nx & $61(57.5)$ \\
\hline N1 & $45(42.5)$ \\
\hline Preoperative M stage & \\
\hline M1a & $36(34.0)$ \\
\hline M1b, 1 bone lesion & $20(18.9)$ \\
\hline M1b, 2 bone lesions & $7(6.6)$ \\
\hline M1b, $\geq 3$ bone lesions & $9(8.5)$ \\
\hline M1b, number not recorded & $34(32.1)$ \\
\hline
\end{tabular}

patients had a bone biopsy to confirm metastases, and the others were assessed by bone scan, magnetic resonance imaging, computed tomography, or positron emission tomography. Recording of the timing of neoadjuvant, adjuvant, and salvage therapies was not consistent across centers, so postoperative PSA measurements could not be interpreted. However, individual clinicians confirmed that all patients received ADT before death, with 11/106 (10.4\%) men undergoing adjuvant and 6/106 (5.7\%) salvage radiation therapy.

The surgical modality of choice was open radical prostatectomy for four out of six centers, with one center performing both open $(27 / 31 ; 87.1 \%)$ and robotic $(4 / 31$; $12.9 \%)$ procedures and one performing all cases robotically

Table 3 - Operative approach, overall complications, operative time, and length of hospital stay stratified by center

\begin{tabular}{|c|c|c|c|c|c|c|}
\hline \multirow[t]{2}{*}{ Center } & \multirow{2}{*}{$\begin{array}{l}\text { Patients } \\
(n)\end{array}$} & \multicolumn{2}{|c|}{ Approach } & \multirow[t]{2}{*}{ Operative time (min) } & \multirow[t]{2}{*}{ Length of stay (d) } & \multirow[t]{2}{*}{ Complications at $90 \mathrm{~d}, n(\%)$} \\
\hline & & Open, $n(\%)$ & Robotic $(n)$ & & & \\
\hline 1 & 31 & $31(100)$ & 0 & $190(164-247)$ & $3(3-5)$ & $4(12.9)$ \\
\hline 2 & 31 & $27(87.1)$ & 4 & $79.5(67-140)$ & $11(9-13)$ & $4(12.9)$ \\
\hline 3 & 25 & $25(100)$ & 0 & $180(156-212.5)$ & $7(6-8)$ & $6(24.0)$ \\
\hline 4 & 11 & $11(100)$ & 0 & $170(160-380)$ & $13(7-19)$ & $6(54.5)$ \\
\hline 5 & 5 & $0(0)$ & 5 & $147(130-180)$ & $3(3-3)$ & $2(40.0)$ \\
\hline 6 & 3 & $3(100)$ & 0 & $159(147-170)$ & $9(7-10)$ & $0(0)$ \\
\hline
\end{tabular}


Table 4 - Postoperative margin status, pathologic stage, and continence for the whole cohort

\begin{tabular}{lc}
\hline & $n(\%)$ \\
\hline Margin status & \\
Positive & $57(54.3)$ \\
Negative & $48(45.7)$ \\
Postoperative T stage & $2(1.9)$ \\
T0/Tx & $21(19.8)$ \\
T2 & $14(13.2)$ \\
T3a & $57(53.8)$ \\
T3b & $12(11.3)$ \\
T4 & \\
Postoperative N stage & $4(3.8)$ \\
Nx & $26(24.5)$ \\
N0 & $76(71.7)$ \\
N1 & $38(64.4)$ \\
Postoperative continence at 90 d & $10(17.0)$ \\
\hline $0-1$ pad (for security) & $11(18.6)$ \\
\hline 1-2 pads (mild incontinence) & \\
\hline 3 pads (moderate/severe incontinence) & \\
\hline
\end{tabular}

(Table 3$)$. The median operative time was approximately $2.5 \mathrm{~h}$ (164.5 $\mathrm{min}$ ), and the US and Swedish centers were the quickest to discharge, with a median length of stay of $3 \mathrm{~d}$ compared to the overall median of $8 \mathrm{~d}$.

Postoperative data are shown in Table 4; 57/105 (54.3\%) cases had positive surgical margins, with missing data for one individual. Some 83/106 (78.3\%) men had at least pT3 disease and the median Gleason score was 8.5 . The median number of nodes removed was 18 (IQR 11-27); 71.7\% (76/ 106) of men had positive nodes and there were four $\mathrm{Nx}$ cases. Continence at $90 \mathrm{~d}$ after radical prostatectomy was recorded in 59/106 (55.7\%) patients, and most (38/59; $64.4 \%$ ) were dry, with only $18.6 \%$ (11/59) suffering moderate/severe incontinence.

Complications at $90 \mathrm{~d}$ were reported by all six centers according to the variables abstracted in the meta-analysis of Tewari et al [16] (Table 5). Most patients had no complications. However, two cases require reoperation before initial hospital discharge, one for pelvic hematoma and one for an infected lymphocele not suitable for percutaneous drainage because of its location. Four men were readmitted to hospital after discharge but did not require reoperation, two for percutaneous drainage of infected lymphoceles, one for intravenous rehydration and antibiotics because of exacerbation of diverticulitis, and one for intravenous rehydration only. Blood transfusions were given to $15 / 106$ (14.2\%) patients. In total, 22/106 (20.8\%) patients had a complication; lymphocele (9/106; 8.5\%), anastomotic leak (7/106; 6.6\%), and wound infection $(5 / 106 ; 4.7 \%)$ occurred most frequently. Of those who suffered complications, 6/22 (27.3\%) had two complications and no man suffered more than two complications (Table 6).

In total, $12 / 106$ (11.3\%) men died from prostate cancer at a median follow-up of $22.8 \mathrm{mo}$, but only one patient died within $1 \mathrm{yr}$ of radical prostatectomy. No man died of other causes. Ten of the 12 deaths (83.3\%) were in men with skeletal metastases at presentation. Kaplan-Meier survival plots for the whole cohort and stratified by M substage are shown in Figure 1. One patient was excluded from this analysis because of missing data on the date of surgery.
Table 5 - Rates of reoperation, readmission, transfusion, and 21 specific complications for the whole cohort

\begin{tabular}{lc}
\hline & $n(\%)$ \\
\hline Reoperation & $2(1.9)$ \\
\hline Readmission & $4(3.8)$ \\
\hline Blood transfusion & $15(14.2)$ \\
\hline latrogenic injury & \\
\hline Vessel & $0(0)$ \\
Obturator nerve & $0(0)$ \\
Ureteral & $1(0.9)$ \\
Bladder & $0(0)$ \\
Rectal & $0(0)$ \\
Other bowel & $0(0)$ \\
\hline Need for ileostomy & $0(0)$ \\
Ileus & $1(0.9)$ \\
\hline Deep vein thrombosis & $1(0.9)$ \\
Pulmonary embolism & $0(0)$ \\
Pneumonia & $0(0)$ \\
Myocardial infarction & $0(0)$ \\
Symptomatic hematoma & $2(1.9)$ \\
\hline Symptomatic lymphocele & $9(8.5)$ \\
\hline Anastomotic leak & $7(6.6)$ \\
Anastomotic stricture & $1(0.9)$ \\
Sepsis & $1(0.9)$ \\
Wound infection & $5(4.7)$ \\
\hline Wound dehiscence & $0(0)$ \\
\hline
\end{tabular}

(A)

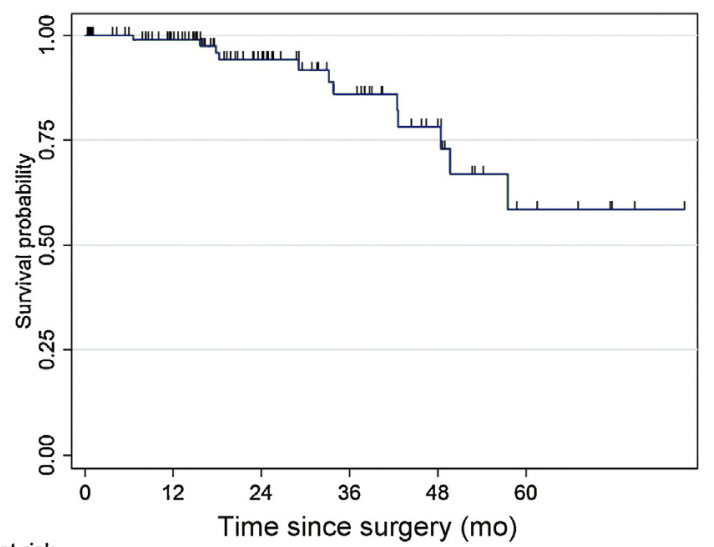

Number at risk

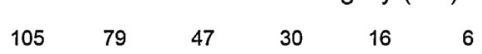

(B)

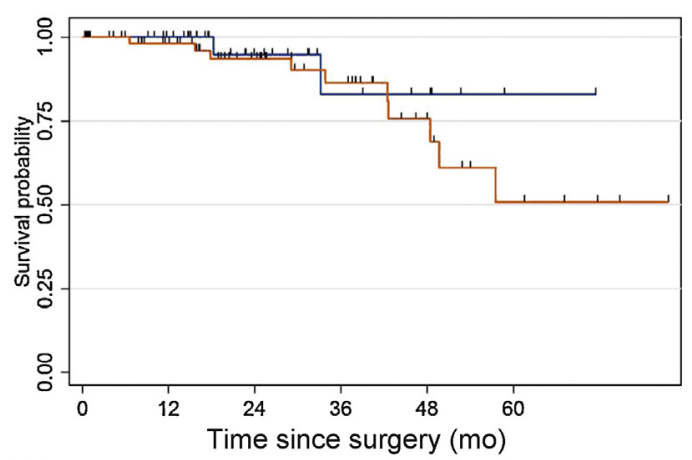

Number at risk

\begin{tabular}{|c|c|c|c|c|c|c|}
\hline \multirow{2}{*}{\multicolumn{2}{|c|}{$\begin{array}{ll}\text { M1a } & 36 \\
\text { M1b } & 69\end{array}$}} & \multirow{2}{*}{$\begin{array}{l}31 \\
48\end{array}$} & 14 & 7 & 5 & 1 \\
\hline & & & 33 & 23 & 11 & 5 \\
\hline & & & & M1a & & M1b \\
\hline
\end{tabular}

Fig. 1 - Prostate cancer survival and overall survival for $(A)$ the whole cohort and (B) patients stratified by $M$ substage. 
Table 6 - Complication occurrence for patients who had at least one complication

\begin{tabular}{|c|c|c|c|c|c|c|c|c|c|}
\hline Patient & $\begin{array}{l}\text { Iatrogenic } \\
\text { ureteral injury }\end{array}$ & Ileus & DVT & Hematoma & Lymphocele & $\begin{array}{l}\text { Anastomotic } \\
\text { leak }\end{array}$ & $\begin{array}{l}\text { Anastomotic } \\
\text { stricture }\end{array}$ & Sepsis & $\begin{array}{l}\text { Wound } \\
\text { infection }\end{array}$ \\
\hline 1 & 0 & 0 & 0 & 0 & 0 & 1 & 0 & 0 & 1 \\
\hline 2 & 0 & 0 & 0 & 0 & 0 & 0 & 0 & 0 & 1 \\
\hline 3 & 0 & 0 & 0 & 0 & 1 & 0 & 0 & 0 & 0 \\
\hline 4 & 0 & 0 & 0 & 0 & 1 & 0 & 0 & 0 & 0 \\
\hline 5 & 0 & 1 & 0 & 0 & 1 & 0 & 0 & 0 & 0 \\
\hline 6 & 0 & 0 & 0 & 0 & 0 & 1 & 0 & 0 & 1 \\
\hline 7 & 0 & 0 & 0 & 0 & 0 & 1 & 0 & 0 & 0 \\
\hline 8 & 0 & 0 & 0 & 0 & 0 & 0 & 0 & 0 & 1 \\
\hline 9 & 0 & 0 & 0 & 0 & 0 & 1 & 0 & 0 & 0 \\
\hline 10 & 0 & 0 & 0 & 0 & 1 & 0 & 0 & 0 & 0 \\
\hline 11 & 0 & 0 & 0 & 0 & 1 & 0 & 0 & 0 & 0 \\
\hline 12 & 0 & 0 & 0 & 1 & 1 & 0 & 0 & 0 & 0 \\
\hline 13 & 0 & 0 & 0 & 0 & 1 & 0 & 0 & 0 & 0 \\
\hline 14 & 0 & 0 & 0 & 0 & 0 & 0 & 1 & 0 & 0 \\
\hline 15 & 0 & 0 & 0 & 1 & 0 & 0 & 0 & 0 & 0 \\
\hline 16 & 0 & 0 & 1 & 0 & 0 & 0 & 0 & 0 & 0 \\
\hline 17 & 0 & 0 & 0 & 0 & 0 & 1 & 0 & 0 & 0 \\
\hline 18 & 0 & 0 & 0 & 0 & 0 & 0 & 0 & 0 & 1 \\
\hline 19 & 0 & 0 & 0 & 0 & 1 & 0 & 0 & 0 & 0 \\
\hline 20 & 0 & 0 & 0 & 0 & 1 & 0 & 0 & 0 & 0 \\
\hline 21 & 0 & 0 & 0 & 0 & 0 & 1 & 0 & 1 & 0 \\
\hline 22 & 1 & 0 & 0 & 0 & 0 & 1 & 0 & 0 & 0 \\
\hline
\end{tabular}

Reverse Kaplan-Meier median follow-up times were 20.7 and 24.1 mo for M1a and M1b survivors, respectively (data not shown). Complication rates were similar in the two subgroups: $\mathrm{M} 1 \mathrm{a}=7 / 36$ (19.4\%) and M1b=15/70 (21.4\%). The 21 individual complications did not obviously differ between the subgroups (data not shown).

\section{Discussion}

Interest is emerging in the possible role of radical intervention in metastatic prostate cancer, but it would be inappropriate to investigate the impact of radical prostatectomy in metastatic prostate cancer without a detailed assessment of its feasibility and safety. We evaluated perioperative and complication outcomes in 106 men with M1 prostate cancer at presentation who were operated on in four countries across six centers. Four-fifths of men suffered no complications and only one patient had an intraoperative injury, suggesting that radical prostatectomy is indeed technically feasible and safe in men with metastatic prostate cancer. Most complications were related to the extended pelvic lymphadenectomy, and prostatic extirpation did not add further significant morbidity.

Heidenreich et al [17] compared patients treated with radical prostatectomy for prostate cancer with skeletal metastases to a control group managed with ADT, and found that cancer-specific survival rates favored those treated operatively ( $95.6 \%$ vs $84.2 \% ; p=0.043$ ), although the study was limited by its short follow-up and selection biases. The overall complication rate was reported as 5/23 (21.7\%), but no comprehensive assessment of all known individual complications was performed. In the current study we assessed the safety of surgery in M1 disease across multiple centers. Although we did not primarily investigate oncologic outcome, we observed similar survival rates to those in the CHAARTED study for patients with low-volume metastatic disease [18]; as expected, we found that men with nonregional nodal metastases fared better oncologically after radical prostatectomy than those with skeletal disease. Several observational studies in men with regional nodal metastases have indicated a survival benefit for surgery, and current European Association of Urology (EAU) guidelines sanction surgery as an optional treatment for N1 prostate cancer in the context of multimodal therapy [19,20]. In fact, a recent survey of EAU members showed that two-thirds of practicing European urologists favor this approach [21].

The overall complication rate of $20.8 \%$ in our study is consistent with that reported for radical prostatectomy for standard indications; Tewari et al [16] completed a large meta-analysis of 400 original articles, examining complication outcomes for 286876 patients, and found overall rates of $8.2-19.4 \%$, the latter being for the 167184 open radical prostatectomies. An open approach was used for more than $90 \%$ of our cases. Thus, again not withstanding our retrospective study design, it appears that there is no general increase in morbidity for surgery in metastatic disease compared with standard indications, as reported in the above meta-analysis. Our readmission (3.8\%), reoperation (1.9\%), and transfusion rates (14.2\%) are also similar to those for open surgeries in the meta-analysis (3.0\%, 2.3\%, and $16.5 \%$, respectively). The mean length of stay was 3.1 and $9.9 \mathrm{~d}$ for open cases in the meta-analysis for US and non-US centers, respectively, with corresponding averages of 3 and $8 \mathrm{~d}$ for our study cohort. Differences in discharge pattern appear to be based on national policy rather than on complications. The length of stay for the one purely robotic center in our study was greater than that reported for 62389 cases in the metaanalysis (average 3 vs $1.4 \mathrm{~d}$ ). Nevertheless, the results demonstrate that a robotic approach is feasible for metastatic cases.

The median lymph-node count of 18 is suggestive of satisfactory extended pelvic lymphadenectomy in our 
series, and the symptomatic lymphocele rate of $8.5 \%$ is comparable to the $9.4 \%$ reported for another series of 434 patients undergoing extended lymphadenectomy [26]. Our anastomotic leak rate of $6.6 \%$ compares favorably with the $10.0 \%$ for patients undergoing open surgery in the Tewari et al meta-analysis [16]. Five of our cases (4.7\%) suffered a wound infection, which is higher than the mean of $2.8 \%$ for open surgery in the meta-analysis, but with so few complications it is difficult to comment on the significance of this. All other specific complications were similar between our cohort and the meta-analysis, leading to the conclusion that radical prostatectomy is safe in men with metastatic prostate cancer, and any extra morbidity over surgery for standard indications can probably be attributed to the extended pelvic lymphadenectomy. Current EAU guidelines recommend extended pelvic lymphadenectomy in all highrisk patients [27], and thus surgery for metastatic disease does not involve greater morbidity than that for high-risk localized prostate cancer.

Four-fifths of our patients had pT3-4 prostate cancer, so our positive margin rate of $54.3 \%$ is not unexpected and not particularly high compared to the $42.6 \%$ observed for pT3 open surgical cases in the Tewari et al [16] meta-analysis. A prospective study for 236 men who underwent open retropubic radical prostatectomy found a 3-mo continence of $50 \%$ [28], so our rate of $64.4 \%$ demonstrates comparable morbidity for radical prostatectomy in metastatic disease.

Our study has limitations: it is a retrospective analysis of data from different centers with differences in surgical decision-making and nebulous selection criteria. In fact, no well-defined selection criteria were followed; rather, the decision to operate was based purely on the clinician's subjective judgment, making this analysis subject to significant selection bias. Metastatic burden was not recorded in many cases, and methods of assessing the burden were not standardized either. Complication rates and other data were obtained via retrospective review of notes, which is less reliable than a prospectively collected data set with a defined study protocol. How and what data were recorded varied between centers, and we relied on the quality of the notekeeping to inform complication recording. Also, the followup is too short to make inferences about the oncologic benefit of surgery, and this paper can only comment on the technical feasibility and safety, and not the oncologic value, of radical prostatectomy in metastatic prostate cancer. Finally, all patients had $\mathrm{CCI}<2$ and all cases were chosen according to surgeon discretion; hence, here is likely to be significant selection bias and these results cannot be generalized to all men with metastatic prostate cancer. The lack of performance status (with only CCI as a surrogate) also represents a limitation, since it is likely these men were fitter than the general population with metastatic disease. In essence, this study demonstrates outcomes in expert hands and meticulously selected patients, and cannot be extrapolated to allcomers with metastatic prostate cancer. However, the study represents the largest and only multi-institutional series to date examining perioperative and complication outcomes across multiple domains after surgery in distant metastatic prostate cancer. Thus, it provides the current best evidence supporting the safety of radical prostatectomy in men with metastatic (M1) prostate cancer, and will help to inform future trials of surgery as radical intervention for select cases.

\section{Conclusions}

Radical prostatectomy for men with locally resectable, distant metastatic prostate cancer appears safe in expert hands for meticulously selected patients. Overall and specific complication rates related to the surgical extirpation are not more frequent than when radical prostatectomy is performed for standard indications, and the use of extended pelvic lymphadenectomy in all of this cohort compared to its selective use in localized/locally advanced prostate cancer accounts for any extra morbidity.

Author contributions: Prasanna Sooriakumaran had full access to all the data in the study and takes responsibility for the integrity of the data and the accuracy of the data analysis.

Study concept and design: Sooriakumaran, Graefen, Wiklund.

Acquisition of data: Karnes, Stief, Montorsi, Hammerer, Beyer, Moschini, Gratzke, Steuber, Suardi, Briganti, Manka, Nyberg, Wiklund, Graefen. Analysis and interpretation of data: Copsey, Dutton, Sooriakumaran. Drafting of the manuscript: Sooriakumaran.

Critical revision of the manuscript for important intellectual content: Graefen, Wiklund.

Statistical analysis: Copsey, Dutton.

Obtaining funding: None.

Administrative, technical, or material support: None.

Supervision: Graefen, Wiklund.

Other (specify): None.

Financial disclosures: Prasanna Sooriakumaran certifies that all conflicts of interest, including specific financial interests and relationships and affiliations relevant to the subject matter or materials discussed in the manuscript (eg, employment/affiliation, grants or funding, consultancies, honoraria, stock ownership or options, expert testimony, royalties, or patents filed, received, or pending), are the following: None.

Funding/Support and role of the sponsor: None.

\section{References}

[1] Center MM, Jemal A, Lortet-Tieulent J, et al. International variation in prostate cancer incidence and mortality rates. Eur Urol 2012; 61:1079-92.

[2] Yossepowitch O, Bianco Jr FJ, Eggener SE, Eastham JA, Scher HI, Scardino PT. The natural history of noncastrate metastatic prostate cancer after radical prostatectomy. Eur Urol 2007;51:940-7.

[3] James ND, Spears MR, Clarke NW, et al. Survival with newly diagnosed metastatic prostate cancer in the "docetaxel era": data from 917 patients in the control arm of the STAMPEDE trial (MRC PR08, CRUK/06/019). Eur Urol 2015;65:1028-38.

[4] Gratzke C, Engel J, Stief CG. Role of radical prostatectomy in clinically non-organ-confined prostate cancer. Curr Urol Rep 2014;15:455.

[5] Mickisch GH, Garin A, van Poppel H, et al. Radical nephrectomy plus interferon-alfa-based immunotherapy compared with interferon alfa alone in metastatic renal-cell carcinoma: a randomised trial. Lancet 2001;358:966-70.

[6] Flanigan RC, Salmon SE, Blumenstein BA, et al. Nephrectomy followed by interferon alfa- $2 \mathrm{~b}$ compared with interferon alfa- $2 \mathrm{~b}$ alone for metastatic renal-cell cancer. N Engl J Med 2001;345:1655-9. 
[7] Bristow RE, Tomacruz RS, Armstrong DK, Trimble EL, Montz FJ. Survival effect of maximal cytoreductive surgery for advanced ovarian carcinoma during the platinum era: a meta-analysis. J Clin Oncol 2002;20:1248-59.

[8] Culp SH, Schellhammer PF, Williams MB. Might men diagnosed with metastatic prostate cancer benefit from definitive treatment of the primary tumor? A SEER-based study. Eur Urol 2014;65:1058-66.

[9] Gratzke C, Engel J, Stief CG. Role of radical prostatectomy in metastatic prostate cancer: data from the Munich Cancer Registry. Eur Urol 2014;66:602-3.

[10] Sooriakumaran P, Nyberg T, Akre O, et al. Comparative effectiveness of radical prostatectomy and radiotherapy in prostate cancer: observational study of mortality outcomes. BMJ 2014;348:g1502.

[11] Psaila B, Lyden D. The metastatic niche: adapting the foreign soil. Nat Rev Cancer 2009;9:285-93.

[12] Ceelen W, Pattyn P, Mareel M. Surgery, wound healing, and metastasis: recent insights and clinical implications. Crit Rev Oncol Hematol 2014;89:16-26.

[13] Hong MK, Macintyre G, Wedge DC, et al. Tracking the origins and drivers of subclonal metastatic expansion in prostate cancer. Nat Commun 2015;6:6605.

[14] Gundem G, Van Loo P, Kremeyer B, et al. The evolutionary history of lethal metastatic prostate cancer. Nature 2015;520:353-7.

[15] Chapin BF, McGuire SE, Aparicio A. Is treatment of the primary tumor in metastatic prostate cancer justified? Eur Urol 2014; 65:1067-8.

[16] Tewari A, Sooriakumaran P, Bloch DA, Seshadri-Kreaden U, Hebert AE, Wiklund P. Positive surgical margin and perioperative complication rates of primary surgical treatments for prostate cancer: a systematic review and meta-analysis comparing retropubic, laparoscopic, and robotic prostatectomy. Eur Urol 2012;62:1-15.
[17] Heidenreich A, Pfister D, Porres D. Cytoreductive radical prostatectomy in patients with prostate cancer and low volume skeletal metastases: results of a feasibility and case-control study. J Urol 2015;193:832-8.

[18] Sweeney C, Chen Y, Carducci M, et al. Impact on overall survival (OS) with chemohormonal therapy versus hormonal therapy for hormone-sensitive newly metastatic prostate cancer ( $\mathrm{mPrCa}$ ): an ECOG-led phase III randomized trial. J Clin Oncol 2014;32 (Suppl 5s):LBA2.

[19] Gakis G, Boorjian SA, Briganti A, et al. The role of radical prostatectomy and lymph node dissection in lymph node-positive prostate cancer: a systematic review of the literature. Eur Urol 2014;66:191-9.

[20] Heidenreich A, Bastian PJ, Bellmunt J, et al. EAU guidelines on prostate cancer. Part II: Treatment of advanced, relapsing, and castration-resistant prostate cancer. Eur Urol 2014;65:467-79.

[21] Surcel CI, Sooriakumaran P, Briganti A, et al. Preferences in the management of high-risk prostate cancer among urologists in Europe: results of a web-based survey. BJU Int 2015;115:571-9.

[26] Musch M, Klevecka V, Roggenbuck U, Kroepfl D. Complications of pelvic lymphadenectomy in 1,380 patients undergoing radical retropubic prostatectomy between 1993 and 2006. J Urol 2008; 179:923-8.

[27] Heidenreich A, Bastian PJ, Bellmunt J, et al. EAU guidelines on prostate cancer. Part 1: screening, diagnosis, and local treatment with curative intent-update 2013. Eur Urol 2014;65:124-37.

[28] Treiyer A, Anheuser P, Butow Z, Steffens J. A single center prospective study: prediction of postoperative general quality of life, potency and continence after radical retropubic prostatectomy. J Urol 2011;185:1681-5. 